Antonio F B. de A Prado

Instituto Nacional de Pesquisas Espaciais 12227-010 São José dos Campos, SP. Brazil prado@dem.inpe.br

\section{Analytical and Numerical Approaches to Study the Gravitational Capture in the Four-Body Problem}

The objective of this paper is to study the problem of gravitational capture in the bicircular restricted four-body problem. A gravitational capture occurs when a massless particle changes its two-body energy around one celestial body from positive to negative without the use of non-gravitational forces. It is mainly studied the effect of the fourth-body included in the dynamics. The Earth-Moon system with the inclusion of the Sun is used for the numerical simulations. The results show the savings obtained in this more realistic model when compared with the more traditional restricted three-body problem model. It is clear that large savings are obtained thanks to the effect of the Sun, if a proper geometry is used for the maneuver.

Keywords: Astrodynamics, gravitational capture, celestial mechanics, restricted four-body problem

\section{Introduction}

The ballistic gravitational capture is a characteristic of some dynamical systems in celestial mechanics, as in the restricted fourbody problem that is considered in this paper. The basic idea is that a spacecraft (or any particle with negligible mass) can change from a hyperbolic orbit with a small positive energy around a celestial body into an elliptic orbit with a small negative energy without the use of any propulsive system. The force responsible for this modification in the orbit of the spacecraft is the gravitational force of the third and the fourth bodies involved in the dynamics. In this way, this force is used as a zero cost control, equivalent to a continuous thrust applied in the spacecraft. One of the most important applications of this property is the construction of trajectories to the Moon.

The application of this phenomenon in spacecraft trajectories is recent in the literature. The first demonstration of this was in Belbruno, 1987. Further studies include Belbruno (1990 and 1992); Krish (1991); Krish, Belbruno and Hollister (1992); Miller and Belbruno (1991); Belbruno and Miller (1990 and 1993). They all studied missions in the Earth-Moon system that use this technique to save fuel during the insertion of the spacecraft in its final orbit around the Moon. Another set of papers that made fundamental contributions in this field, also with the main objective of constructing real trajectories in the Earth-Moon system, are those of Yamakawa, Kawaguchi, Ishii and Matsuo (1992 and 1993), Yamakawa (1992) and Kawaguchi (2000). The first real application of a ballistic capture transfer was made during an emergency in a Japanese spacecraft (Belbruno and Miller, 1990). After that, some studies that consider the time required for this transfer appeared in the literature. Examples of this approach can be found in the papers by Vieira-Neto and Prado (1995 and 1998). An extension of the dynamical model to consider the effects of the eccentricity of the primaries is also available in the literature (Vieira-Neto and Prado, 1996; Vieira-Neto, 1999). A study of this problem, from the perspective of invariant manifolds, was developed by Belbruno (1994). An application for a mission to Europa is shown in Sweetser (1997).

Looking in the literature related to the weak stability boundaries, it is possible to see that there are several definitions of ballistic

Presented at XI DINAME - International Symposium on Dynamic Problems of Mechanics, February 28th - March 4th, 2005, Ouro Preto. MG. Brazil.

Paper accepted: June, 2005. Technical Editor: José Roberto de França Arruda. gravitational capture, depending on the dynamical system considered.

Those differences exist to account for the different behavior of the systems. In the restricted four-body problem, the system considered in the present paper, ballistic gravitational capture is assumed to occur when the massless particle stays close to the second primary (the Moon) of the system for some time. A permanent capture is not required, because an impulsive maneuver will be performed to complete the maneuver.

For the practical purposes of studying spacecraft trajectories, the majority of the papers available in the literature study this problem looking in the behavior of the two-body energy of the spacecraft with respect to the Moon. A quantity called $C_{3}$ (that is twice the total energy of a two-body system) is defined, with respect to the closer primary, by $C_{3}=V^{2}-2 \mu / r$, where $V$ is the velocity of the spacecraft relative to the closest primary, $r$ is the distance of the spacecraft from this primary and $\mu$ is the dimensionless gravitational parameter of the primary considered. From the value of $C_{3}$ it is possible to know if the orbit is elliptical $\left(C_{3}<0\right)$, parabolic $\left(C_{3}=0\right)$ or hyperbolic $\left(C_{3}>0\right)$ with respect to the Moon. Based upon this definition, it is possible to see that the value of $C_{3}$ is related to the velocity variation $(\Delta V)$ needed to insert the spacecraft in its final orbit around the Moon. In the case of a spacecraft approaching the Moon, it is possible to use the gravitational force of the Earth to lower the value of $C_{3}$ with respect to the Moon, so the fuel consumption required to complete this maneuver is reduced. In that way, the search for trajectories that arrives at the Moon with the maximum possible value for the reduction of $C_{3}$ is very important. In the majority of the studies relative to this problem, a numerical approach of verifying the values of $C_{3}$ during the trajectory is used to identify useful trajectories. If there is a change of sign in $C_{3}$ from negative (closed trajectory) to positive (open trajectory) when leaving the Moon, it means that a ballistic gravitational capture occurs in the positive sense of time and this particular trajectory can be used to reduce the amount of fuel in an Earth-Moon transfer. The present paper has the goal of developing analytical equations to estimate the effects of the fourth body in the reduction of $C_{3}$. This paper was first presented at the XI International Symposium on Dynamic Problems of Mechanics - XI DINAME.

\section{Mathematical Model and Some Properties}

The model used in this paper is the planar bi-circular restricted four-body problem. This model assumes that two main bodies $\left(M_{1}\right.$ and $M_{2}$ ) are orbiting their common center of mass in circular 
Keplerian orbits and a third body $\left(M_{3}\right)$ is orbiting these two primaries describing circular orbits around the center of mass of the first two primaries. Then, it is desired to study the motion of a fourth body, $M_{4}$, that is supposed to stay in the plane of the motion of the other three bodies and that is affected by all of them, but it does not affect their motion (Szebehely, 1967). The standard canonical system of units associated with this model is used (the unit of distance is the distance $M_{1}-M_{2}$ and the unit of time is chosen such that the period of the motion of $\mathrm{M}_{2}$ around $M_{1}$ is $2 \pi$ ). Under this model, the equations of motion of the massless particle, in the inertial reference system, are:

$$
\begin{gathered}
\ddot{x}=-\mu_{E} \frac{\left(x-x_{E}\right)}{r_{1}^{3}}-\mu_{M} \frac{\left(x-x_{M}\right)}{r_{2}^{3}}-\mu_{S} \frac{\left(x-x_{S}\right)}{r_{3}^{3}} \\
\ddot{y}=-\mu_{E} \frac{\left(y-y_{E}\right)}{r_{1}^{3}}-\mu_{M} \frac{\left(y-y_{M}\right)}{r_{2}^{3}}-\mu_{S} \frac{\left(y-y_{S}\right)}{r_{3}^{3}} \\
r_{1}=\sqrt{\left(x-x_{E}\right)^{2}+\left(y-y_{E}\right)^{2}} \\
r_{2}=\sqrt{\left(x-x_{M}\right)^{2}+\left(y-y_{M}\right)^{2}} \\
r_{3}=\sqrt{\left(x-x_{S}\right)^{2}+\left(y-y_{S}\right)^{2}} \\
x_{E}=-\mu_{M} \cos (t) \\
y_{E}=-\mu_{M} \sin (t) \\
x_{M}=\mu_{E} \cos (t) \\
y_{M}=\mu_{E} \sin (t) \\
x_{S}=R_{S} \cos (\psi) \\
y_{S}=R_{S} \sin (\psi) \\
\psi=\psi_{0}+\omega_{S} t
\end{gathered}
$$

where $x, y$ are the coordinates of the massless particle, $\mu_{E}$ (0.9878715), $\mu_{M}(0.0121285), \mu_{S}(328900.48)$ are the gravitational parameters of the Earth, Moon and Sun, respectively, $\left(x_{E}, y_{E}\right)\left(x_{M}, y_{M}\right)$ $\left(x_{S}, y_{S}\right)$ are the coordinates of the Earth, Moon and Sun, respectively, $r_{1}, r_{2}, r_{3}$ are the distances between the massless particle and the Earth, Moon and Sun, respectively, $R_{S}(389.1723985)$ is the distance between the Sun and the origin of the reference system, $\omega_{S}$ $(0.07480133)$ is the angular velocity of the Sun, $t$ is the time. Figure 1 shows a sketch of the system considered.

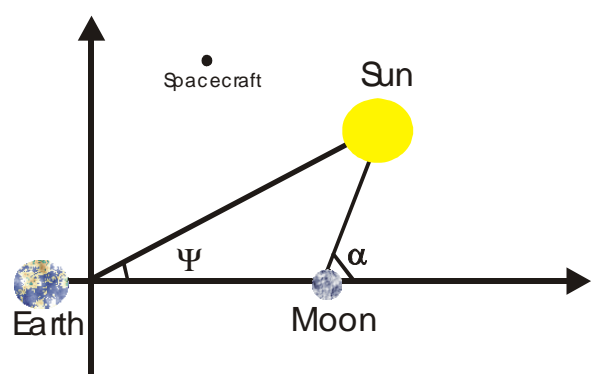

Figure 1. Restricted four-body model (Cartesian Coordinates).

\section{The Gravitational Capture}

To define the gravitational capture it is necessary to use a few basic concepts from the two-body celestial mechanics. Those concepts are:

a) Closed orbit: a spacecraft in an orbit around a central body is in a closed orbit if its velocity is not large enough to escape from the central body. It remains always inside a sphere centered in the central body;

b) Open orbit: a spacecraft in an orbit around a central body is in an open orbit if its velocity is large enough to escape from the central body. In this case the spacecraft can go to infinity, no matter what is its initial position.

To identify the type of orbit of the spacecraft it is possible to use the definition of the two-body energy $(E)$ of a massless particle orbiting a central body. The equation is $E=V^{2} / 2-\mu / r$, where $V$ is the velocity of the spacecraft relative to the central body, $\mu$ is the gravitational parameter of the central body and $r$ is the distance between the spacecraft and the central body.

With this definition it is possible to say that the spacecraft is in an open orbit if its energy is positive and that it is in a closed orbit if its energy is negative. In the two-body problem this energy remains constant and it is necessary to apply an external force to change it. This energy is no longer constant in the bi-circular four-body problem. Then, for some initial conditions, a spacecraft can alternate the sign of its energy from positive to negative or from negative to positive. When the variation is from positive to negative the maneuver is called a "gravitational capture", to emphasize that the spacecraft was captured by gravitational forces only, with no use of an external force, like the thrust of an engine. The opposite situation, when the energy changes from negative to positive is called a "gravitational escape". In the bi-circular four-body problem and in the restricted three-body problem there is no permanent gravitational capture. If the energy changes from positive to negative, it will change back to positive in the future.

One of the most important applications of the gravitational capture can be found in trajectories to the Moon. The concept of gravitational capture is used together with the basic ideas of the gravity-assisted maneuver and the bi-elliptic transfer orbit to generate a trajectory that requires a fuel consumption smaller than the one required by the Hohmann transfer. This maneuver consists of the following steps: i) the spacecraft is launched from an initial circular orbit with radius $r_{0}$ to an elliptic orbit that crosses the Moon's path; ii) a Swing-By with the Moon is used to increase the apoapsis of the elliptic orbit. This step completes the first part of the bi-elliptic transfer, with some savings in $\Delta V$ due to the energy gained from the Swing-By; iii) With the spacecraft at the apoapsis, a second very small impulse is applied to rise the periapsis to the Earth-Moon distance. Solar effects can reduce even more the magnitude of this impulse; iv) The transfer is completed with the gravitational capture of the spacecraft by the Moon.

\section{Forces Involved in the Dynamics}

To understand better the physical reasons of this phenomenon, it is useful to calculate the forces acting over the massless particle. Figure 2 shows the gravitational force $\vec{F}_{g}$ of the Earth acting in a spacecraft $\mathrm{M}_{3}$ that is approaching the Moon and Fig. 3 shows the gravitational force of the Sun and the centrifugal force acting in the same situation. There is also the Coriolis force, given by $-2 \vec{\omega}_{E-M} \times \vec{v}$, where $\overrightarrow{\mathrm{V}}$ is the velocity of the spacecraft. This force is not analyzed in detail because the main idea of this paper is to explain the ballistic gravitational capture as a result of perturbative forces acting in this direction and the Coriolis force acts 
perpendicular to the direction of motion of the spacecraft all the time. In this way, it does not contribute to the phenomenon studied here. The direction $\vec{r}$ points directly to the center of the Moon and the direction $\vec{p}$ is perpendicular to $\vec{r}$, pointing in the counterclockwise direction. The distance between the spacecraft and the Earth is d, the angle formed by the line connecting the Earth to the spacecraft and the direction $\vec{r}$ is $\gamma$. The angle $\phi$ is used to define instantaneously the direction $\vec{r}$. From geometrical considerations shown in more detail in Prado (2002), it is possible to write for the gravitational force:

$$
\begin{gathered}
\vec{F}_{g}=\frac{(1-\mu)(r+\cos \phi)}{d^{3}} \vec{r}+\frac{(1-\mu) \sin \phi}{d} \vec{p}= \\
=\frac{(1-\mu)(r+\cos \phi)}{\left(1+r^{2}+2 r \cos \phi\right)^{3 / 2}} \vec{r}+\frac{(1-\mu) \sin \phi}{\left(1+r^{2}+2 r \cos \phi\right)^{1 / 2}} \vec{p}
\end{gathered}
$$

For the centrifugal force the expression is (Prado, 2002):

$$
\vec{F}_{c e}=-[r+(1-\mu) \cos \phi] \vec{r}+(\mu-1) \sin \phi \vec{p}
$$

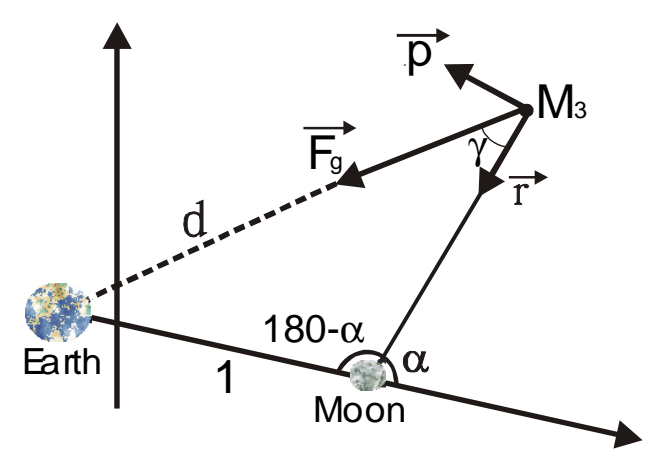

Figure 2. Gravitational force of the Earth.

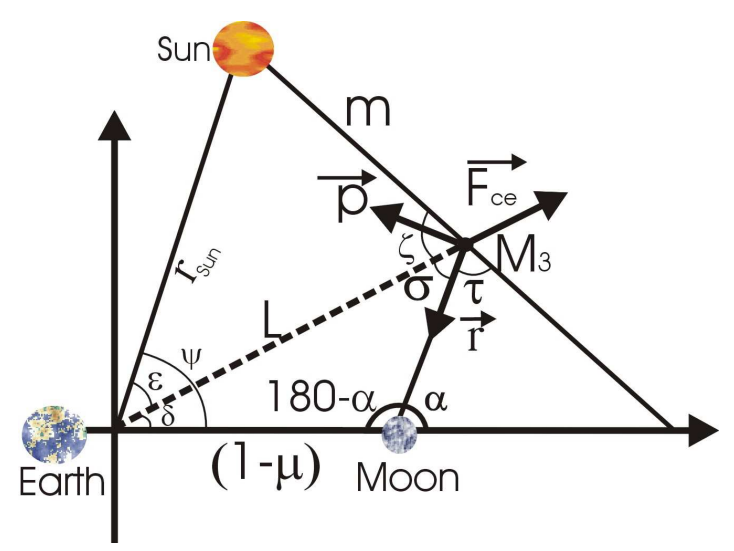

Figure 3. The gravitational force of the Sun and the centrifugal force.

Now, it is necessary to develop an equivalent equation for the gravitational force of the Sun $\left(\vec{F}_{S}\right)$. From Fig. 3 , it is possible to find the following relations, where $\vec{F}_{S r}$ stands for the radial component and $\vec{F}_{S p}$ stands for the perpendicular component:

$$
\vec{F}_{S r}=-\frac{\mu_{s u n} \cos \tau}{m^{2}} \vec{r}, \quad \vec{F}_{S p}=-\frac{\mu_{s u n} \sin \tau}{m^{2}} \vec{p}
$$

$$
\begin{gathered}
R_{\text {sun }}^{2}=L^{2}+m^{2}-2 L m \cos \zeta \Rightarrow \cos \zeta=\frac{R_{\text {sun }}^{2}-L^{2}-m^{2}}{-2 L m} \\
\tau=\pi-\sigma-\zeta \\
\delta=\alpha-\sigma \\
\mathcal{E}=\psi-\delta=\psi-\alpha+\sigma \\
m^{2}=L^{2}+R_{\text {sun }}^{2}-2 L R_{\text {sun }} \cos \varepsilon
\end{gathered}
$$

Those equations can be used to find analytical equations for the radial and perpendicular components of the gravitational force of the Sun.

During the approach phase, when the spacecraft is close to the Moon, the force that dominates the dynamics is due to the central body (the Moon). All others forces are perturbations on the motion of the massless particle. In the model considered here, the perturbations are due to the gravitational force of the Earth and the Sun and the centrifugal force due to the rotation of the system. In that way, an approach to understand the behavior of the perturbing forces is to study the components of each force during the approach phase. This study is performed in Prado (2002), which shows an equation that relates the reduction of $C_{3}$ with the integral of the forces over the time.

\section{Analytical Analyses of the Forces}

The next step of this research is to use the analytical expressions derived in Prado (2002) for the effects of the gravitational force of the Earth and the centrifugal force and to derive an equivalent equation for the gravitational force of the Sun, in order to obtain an estimate of the effects of the forces studied. The main idea is to estimate the potential of the field around the Moon to reduce the value of the $C_{3}$ due to the Earth and the Sun and not to make predictions for a single trajectory. The analytical equations to measure the effects of this perturbation are derived under the assumption that the trajectory followed by the spacecraft is an idealized trajectory that does not deviate from the radial direction. The real trajectories are not radial, as can be seen in the references shown in this paper, but the equations derived under this assumption can be used to: i) estimate the values of the possible reductions in the value of $C_{3}$; ii) show the existence of directions of motion that results in larger reductions of $C_{3}$, so mapping analytically the decelerating field that exists in the neighborhood of the Moon; iii) estimate the effects of the periapsis distance and the size of the sphere of capture, since the equations derived are explicitly functions of those parameters; iv) to study the effect of the fourth body in the savings obtained in the gravitational capture. Another justification for the radial trajectories used to derive the equations is that the reduction of $C_{3}$ is a result of the effects of the forces in time during the whole trajectory and, even for trajectories that shows several loops before arriving at the periapsis, during most of the time the trajectory can be seen as composed by a set of trajectories close to radial.

For the derivation performed here, the component measured is the radial, because this is the direction of motion under the assumption used here. Then, assuming that the spacecraft is in freefall (subject only to the gravitational and centrifugal forces) traveling with zero energy (parabolic trajectory) and that the trajectories do not deviate from a straight line, the result is:

$$
\text { Total energy }=E=0=\frac{1}{2} V^{2}-\frac{\mu}{r} \Rightarrow V=\sqrt{\frac{2 \mu}{r}}=\frac{d s}{d t}
$$


Where $d s$ is the space traveled by the particle during the time $d t$. To obtain the integral of the effect of the perturbing forces with respect to time, it is possible to perform the calculations in terms of the radial distance, by making the substitution:

$$
\int_{t_{0}}^{t_{f}} F d t=\int_{S_{0}}^{s_{f}}(F / V) d s=\int_{r_{\text {min }}}^{r_{\text {max }}}(F / V) d r
$$

The extreme points of the integration changes position $\left(S_{0}\right.$ by $r_{\min }$ and $S_{f}$ by $r_{\max }$ ) here and in all the following integrations to take into account that the positive sense of the radial direction points towards the Moon. Since the spacecraft is assumed to approach the Moon on a radial trajectory the result $\phi=\alpha=\beta$ is valid, and the variable $\alpha$ is used as the independent parameter. Then, for the radial component of the Earth's gravity, up to the first order, the integral is (Prado, 2002):

$$
\begin{aligned}
& F_{1}(\alpha)=\left[\begin{array}{l}
\frac{(1-\mu)(q+\cos \alpha)}{(2 \mu / q)^{1 / 2}\left(1+q^{2}+2 q \cos \alpha\right)^{3 / 2}} r+(1-\mu) \\
\left(-\frac{3(q+\cos \alpha)^{2}}{(2 \mu / q)^{1 / 2}\left(1+q^{2}+2 q \cos \alpha\right)^{5 / 2}}+\right.
\end{array}\right. \\
& \left.\left.+\frac{\mu(3 q+\cos \alpha)}{q^{2}(2 \mu / q)^{3 / 2}\left(1+q^{2}+2 q \cos \alpha\right)^{3 / 2}}\right)\left(\frac{r^{2}}{2}-q r\right)\right]_{r_{\text {min }}}^{r_{\text {max }}}
\end{aligned}
$$

Using the values $r_{\min }=1838 / 384400(100 \mathrm{~km}$ above the lunar surface), $r_{\max }=100000 / 384400(100000 \mathrm{~km}$ above the lunar surface, the usual value for the sphere of capture of the Moon in the ballistic gravitational capture studies), $\mu=0.0121$ (Earth-Moon system) and $q=\left(r_{\text {min }}+r_{\text {max }}\right) / 2$ (the medium point of the trajectory) the first order equation obtained is (Prado, 2002):

$$
F_{1}^{1}(\alpha)=(0.0782+0.5902 \cos (\alpha))(1.0176+0.2649 \cos (\alpha))^{-1.5}
$$

The equivalent equation for the second order expansion is shown below, since in this form it is not too large (Prado, 2002):

\section{$F_{1}(\alpha)=(0.28360 .0170-0.07361 .0175+0.2649 \cos \alpha)+$}

$+0.3076(1.0175+0.2649 \cos \alpha)^{2}+0.0680 \cos \alpha-0.0847(1.0175+$

$+0.2649 \cos \alpha) \cos \alpha+2(1.0175+0.2649 \cos \alpha)^{2} \cos \alpha+$

$+0.0168 \cos 2 \alpha-0.064(1.0175+0.2649 \cos \alpha) \cos 2 \alpha+0.0212 \cos 3 \alpha))$

$(1.0175+0.2649 \cos \alpha)^{-7 / 2}$

For the radial component of the centrifugal force, the integral is:

$$
\begin{aligned}
& r_{\max }\left(F_{c e} / V\right) d s=\int_{r_{\text {min }}}^{r_{\text {max }}}((\mu-1) \cos \alpha+r)(2 \mu / r)^{-1 / 2} d r= \\
& \int_{r_{\text {min }}}=\left[\left(-0.4 r^{2}+\frac{2}{3} r(\mu-1) \cos \alpha\right)(2 \mu / r)^{-1 / 2}\right]_{r_{\text {min }}}^{r_{\max }}
\end{aligned}
$$

Using the same values used in the above situation for the variables, this last equation can be reduced to:

$F_{2}(\alpha)=-0.0887-0.5603 \cos \alpha$

Repeating the process for the gravitational force due to the Sun, we have:

$$
\begin{aligned}
& F_{3}^{1}(\alpha)=\left[\left(\frac{\mu_{\text {sun }}\left(q+\cos \alpha-\mu \cos \alpha-r_{\text {sun }} \cos (\alpha-\psi)\right)}{\sqrt{2 \mu / q}\left(1+q^{2}+r_{\text {sun }}^{2}-2 \mu+\mu^{2}+2 q(1-\mu) \cos \alpha-2 q r_{\text {sun }} \cos (\alpha-\psi)-2 r_{\text {sun }} \cos \psi+2 r_{\text {sun }} \mu \cos \psi\right)^{3 / 2}}\right) r\right]_{r_{\text {min }}}^{r_{\text {max }}} \\
& +\left[\left(-\frac{3\left(2 q+2(1-\mu) \cos \alpha-2 r_{\text {sun }} \cos (\alpha-\psi)\right)\left(q+\cos \alpha-\mu \cos \alpha-r_{\text {sun }} \cos (\alpha-\psi)\right)}{\sqrt{2 \mu / q}\left(1+q^{2}+r_{\text {sun }}^{2}-2 \mu+\mu^{2}+2 q(1-\mu) \cos \alpha-2 q r_{\text {sun }} \cos (\alpha-\psi)-2 r_{\text {sun }} \cos \psi+2 r_{\text {sun }} \mu \cos \psi\right)^{5 / 2}}+\right.\right. \\
& \left.\left.+\frac{\sqrt{q / \mu}+\sqrt{q / \mu}\left(\frac{\left(q+\cos \alpha-\mu \cos \alpha-r_{\text {sun }} \cos (\alpha-\psi)\right)}{2 q}\right)}{\left(1+q^{2}+r_{\text {sun }}^{2}-2 \mu+\mu^{2}+2 q(1-\mu) \cos \alpha-2 q r_{\text {sun }} \cos (\alpha-\psi)-2 r_{\text {sun }} \cos \psi+2 r_{\text {sun }} \mu \cos \psi\right)^{3 / 2}}\right)\left(\frac{\mu_{\text {sun }}}{\sqrt{2}}\right)\left(\frac{r^{2}}{2}-q r\right)\right]_{r_{\text {min }}}^{r_{\max }}
\end{aligned}
$$

and, using the numerical values as done before:

$$
F_{3}^{1}(\alpha)=\frac{A}{B / C}
$$

\section{where}

$A=0.0377(151454+0.2617 \cos \alpha-103.1020 \cos (\alpha-\psi)-768.9220 \cos \psi)^{1 / 2}$

$(-82.3068-268.9200 \cos \alpha-65.7260 \cos (2 \alpha)-4.2907 \cos (3 \alpha)-$

$-119.2110 \cos (\alpha-2 \psi)+6.5904 \cos (3 \alpha-2 \psi)+0.5753 \cos (4 \alpha-2 \psi)+$

$+50403.4000 \cos (\alpha-\psi)+1.7363 \cos (2 \alpha-2 \psi)+$

$+12832.6000 \cos (2 \alpha-\psi)+845.1080 \cos (3 \alpha-\psi)-$

$-0.0029 \cos (4 \alpha-\psi)+12832.9000 \cos (\psi)-32.0000 \cos (2 \psi)+$

$+845.5000 \cos (\alpha+\psi)+0.0842 \cos (2 \alpha+\psi)+$

$+0.0054 \cos (3 \alpha+\psi)-2.1454 \cos (\alpha+2 \psi))$
$B=\left[(3.7960+\cos \alpha)^{2}(-196.9700-0.0003 \cos \alpha+0.1341 \cos (\alpha-\psi)+\cos \psi)^{3}\right]-$ $-0.2845(151454+0.2617 \cos \alpha-103.1020 \cos (\alpha-\psi)-768.9220 \cos \psi)^{1 / 2}$ $(15.4477+37.9366 \cos \alpha+8,8588 \cos (2 \alpha)+0.5684 \cos (3 \alpha)+19.2626 \cos (\alpha-2 \psi)+$ $+2.5985 \cos (3 \alpha-2 \psi)+0.1524 \cos (4 \alpha-2 \psi-6676.6500 \cos (\alpha-\psi))+$ $+13.4051 \cos (2 \alpha-2 \psi)-1699.9300 \cos (2 \alpha-\psi)-111.9640 \cos (3 \alpha-\psi)-$ $-0.0008 \cos (4 \alpha-\psi)-1699.9200 \cos (\psi)+4.4675 \cos (2 \psi)-$

$-112.0040 \cos (\alpha+\psi)-0.0115 \cos (2 \alpha+\psi)-$

$-0.0007 \cos (3 \alpha+\psi)+0.2842 \cos (\alpha+2 \psi))$

$C=\left[(3.7960+\cos \alpha)^{2}(-196.9700-0.0003 \cos \alpha+0.1341 \cos (\alpha-\psi)+\cos \psi)^{3}\right](32)$

The second order equations are too large to be shown and it also has a small contribution compared with the other forces. It is clear 
that the best result, regarding capture, occurs for $\alpha=\psi$, that means that the spacecraft is aligned with the Sun, what is an expected result. Figure 4 shows the effects of the gravitational force of the Sun as a function of $\alpha$ and $\psi$.

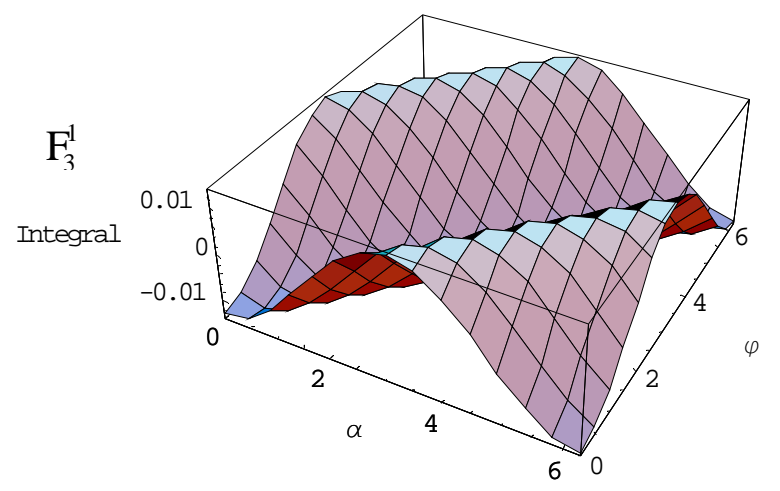

Figure 4. Effects of the gravitational force of the Sun as a function of $\alpha$ and $\psi$.

Adding the radial effects of all the forces, the equation for the resultant force in the radial direction is obtained. This force will be called $F_{r}(\alpha)$. All the forces are plotted as a function of $\alpha$ in Fig. 5, for the case where $\psi=0$. The numbers represents: 1 for the gravitational force due to the Earth; 2 for the centrifugal force; 3 for the gravitational force due to the Sun; 4 for the resultant force. From those results, it is clear that the integral of the total effect is always negative, which means that the spacecraft always has its velocity reduced by the perturbation. It is never increased. There are two points where the integral of the effect is null, which means that the two perturbing forces acting on the spacecraft cancel each other and it travels as if there were no perturbations at all. In this figure it is also possible to obtain the best point to perform the ballistic gravitational capture. This point is at $\alpha=180^{\circ}$, which has the strongest accumulated effect for the resultant force. Figure 6 shows the perturbation of the fourth body $F_{4 b}(\alpha)$ in more detail, for the case where $\psi=0$. Figure 7 shows the resultant forces acting in the motion of the spacecraft including and excluding the Sun. It is clear that the Sun helps to increase the effect of slowing down the spacecraft in an amount of the order of $3 \%$.

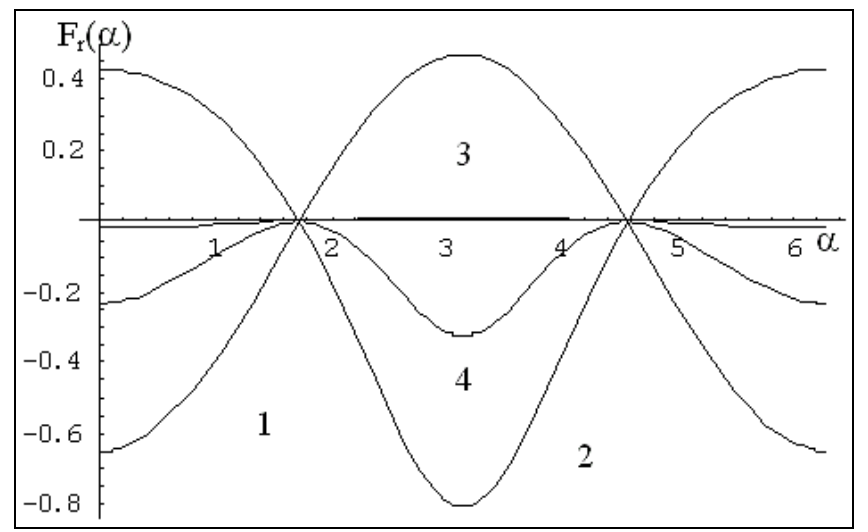

Figure 5. Integral of the disturbing radial forces (canonical units) vs. $\alpha$ (rad) for $\psi=0$.

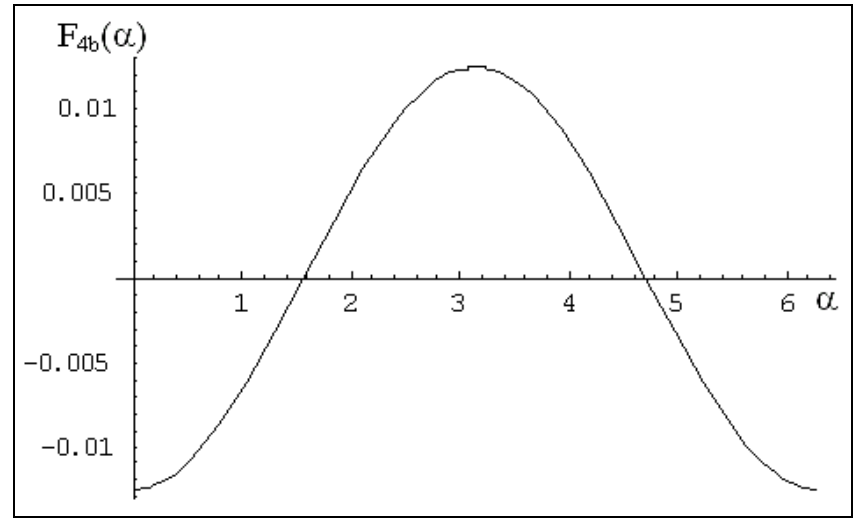

Figure 6. Effects of the gravitational force of the Sun as a function of $\alpha$ for $\psi=0$.

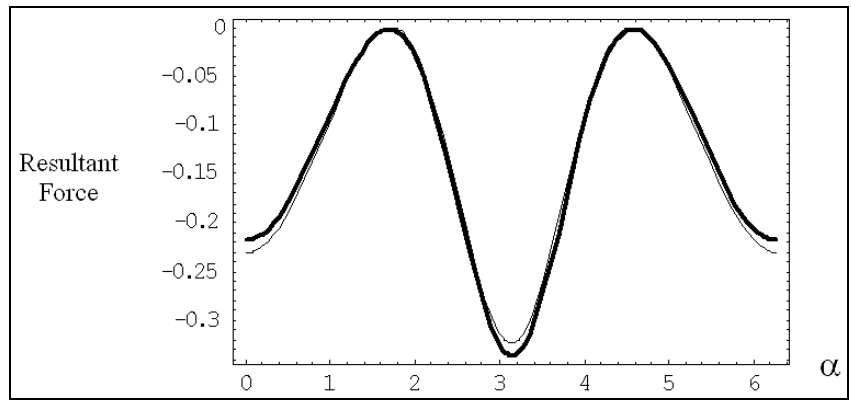

Figure 7. Integral of the resultant force including the Sun (dark line) and excluding the Sun.

\section{Numerical Results}

To make a numerical study of the "gravitational captures" we studied this problem under several different initial conditions. The assumptions made for the numerical examples presented are:

i) The system of primaries used is the Earth-Moon-Sun system (for the bi-circular restricted four-body problem) and the EarthMoon system (for the restricted three-body problem);

ii) The motion is planar everywhere, so the Moon and the Sun are assumed to be in coplanar orbits;

iii) The starting point of each trajectory is $100 \mathrm{~km}$ from the surface of the Moon ( $r_{p}$ from the center of the Moon). Then, to specify the initial position completely it is necessary to give the value of one more variable. The variable used is the angle $\alpha$, an angle measured from the Earth-Moon line, in the counter-clock-wise direction and starting in the side opposite to the Earth (see Fig. 8);

iv) The magnitude of the initial velocity is calculated from a given value of $C 3=2 E=V^{2}-2 \mu / r$, where $E$ is the two-body energy of the spacecraft with respect to the Moon, $V$ is the velocity of the spacecraft, $\mu$ is the gravitational parameter of the Moon and $\mathrm{r}$ is the distance between the spacecraft and the center of the Moon. The direction of the velocity is assumed to be perpendicular to the line spacecraft-center of the Moon and pointing to the counter-clockwise direction for a direct orbit and to the clock-wise direction for a retrograde orbit (see Fig. 8);

v) To consider that an escape occurred, we request, following the conditions used by Yamakawa (1992), that the spacecraft reaches a distance of $100000 \mathrm{~km}$ ( 0.26 canonical units) from the center of the Moon in a time shorter than 50 days. Figure 8 shows the point $\mathrm{P}$ where the escape occurs. The angle that specifies this point is called the "entry position angle" and it is designated with the letter $\beta$. 


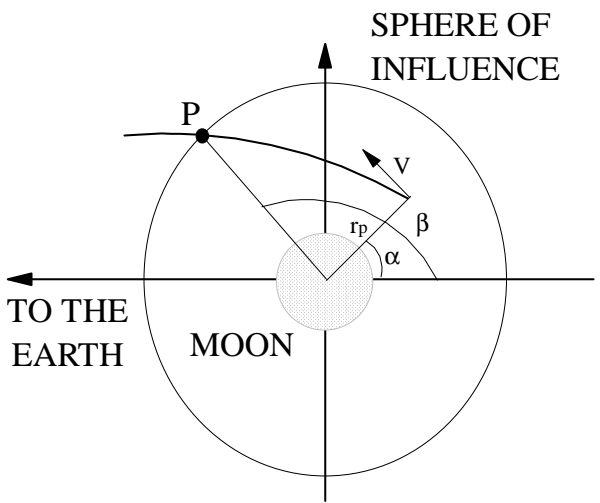

Figure 8. Variables to specify the initial conditions of the spacecraft.

Then, for each initial position the trajectories were numerically integrated backward in time. Every escape in backward time corresponds to a "gravitational capture" in forward time. The results are organized and plotted in the next figure. Figure 9 shows the minimum value of $C 3$ (so, maximum savings obtained in the maneuver) plotted against the angle $\alpha$ in degrees in the horizontal axis. The results are shown for the bi-circular restricted four-body problem (BC4B) and for the restricted three-body problem (RP3B) as a reference. The plots are made for a fixed value of $\psi$, which is shown in every plot.
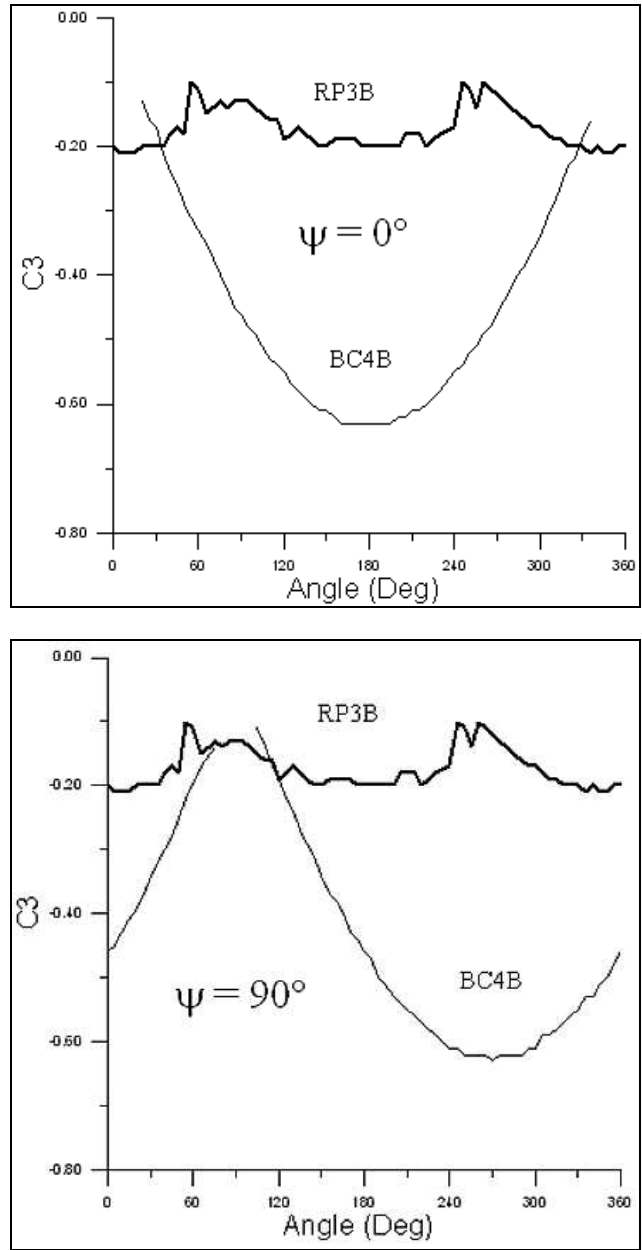

Figure 9. Savings obtained in the bi-circular four body and restricted three-body problem.
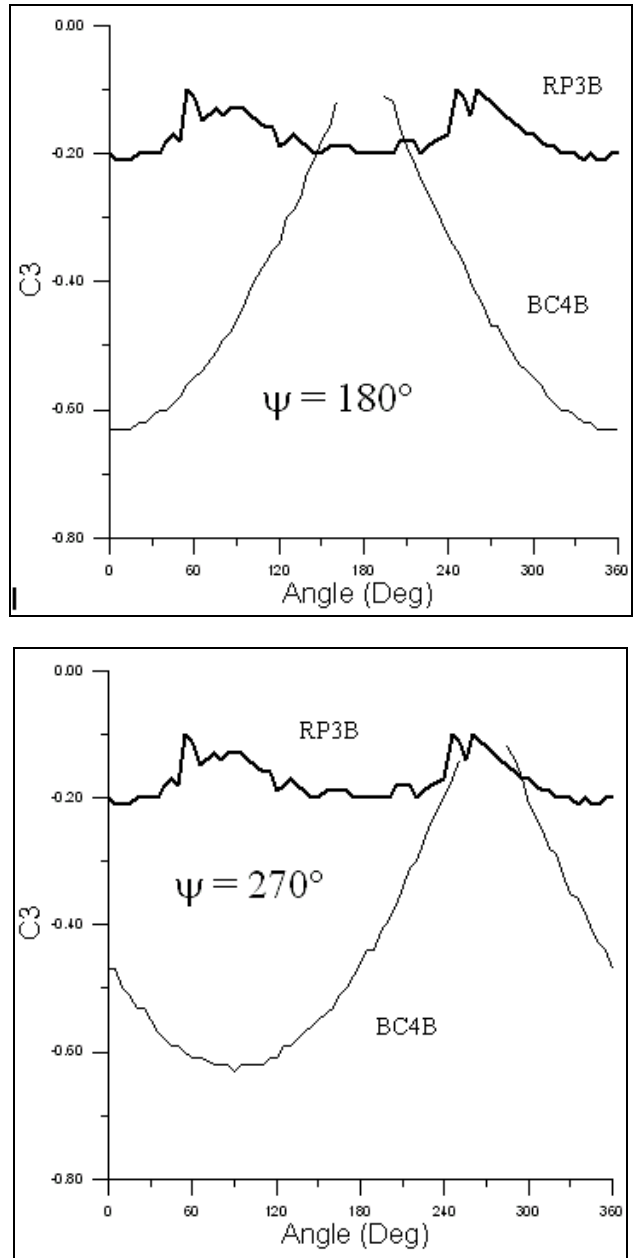

Figure 9. (Continued).

The results show that the savings obtained under the bi-circular restricted four-body problem is very dependent on the initial position of the Sun. This is expected, because since the Sun describes a circular orbit, it can be accelerating or reducing the speed of the spacecraft during its approach to the Moon. In general, the savings can go from a maximum of around -0.62 (about three times the value obtained under the restricted three-body model) up to values close to zero. All the plots also show that there are some regions ( $\psi$ close to $\alpha$ ) where the maneuver is not possible (discontinuities of the plot).

\section{Conclusions}

This paper had the main goal of studying the ballistic gravitational capture problem under the model given by the restricted four-body problem. It showed an explanation of the phenomenon based in the calculation of the forces involved in the dynamics as a function of time and in its integration with respect to time. It also derived analytical equations to study the effect of the fourth body, under the assumption of radial motion. There are three forces that act as disturbing forces in the direction of motion: the gravitational forces due to the Earth and the Sun and the centrifugal force. These forces can slow down the motion of the spacecraft, working opposite to its motion. This is equivalent to applying a continuous propulsion force against the motion of the spacecraft. In the radial direction the gravitational force due to the Earth and the centrifugal force work in opposite directions, but the resultant force 
always works against the motion of the spacecraft, with the exception of two points where they cancel each other. Understanding these behaviors explains why a particle with a velocity slower than the escape velocity can escape from the Moon. The results also showed that the inclusion of the Sun in the dynamics could increase by about $3 \%$ the effects of the forces. The numerical results showed that large savings can be obtained under the bi-circular four-body problem when compared to the restricted three-body problem. In order to obtain those savings, it is necessary to find a proper geometry to start the maneuver. If this is not done, the savings can be reduced or even disappear. Those results are useful to design trajectories to the Moon.

\section{Acknowledgments}

The author is grateful to the Foundation to Support Research in the São Paulo State (FAPESP) for the research grant received under Contract 03/03262-4 and to CNPq (National Council for Scientific and Technological Development) - Brazil for the contract 300828/2003-9.

\section{References}

Belbruno, E.A., "Lunar Capture Orbits, a Method of Constructing Earth Moon Trajectories and the Lunar Gas Mission", AIAA-87-1054. In: 19th AIAA/DGLR/JSASS International Electric Propulsion Conference, Colorado Springs, Colorado, May, 1987.

Belbruno, E.A., "Examples of the Nonlinear Dynamics of Ballistic Capture and Escape in the Earth-Moon System", AIAA-90-2896. In: AIAA Astrodynamics Conference, Portland, Oregon, Aug. 1990.

Belbruno, E.A., "Ballistic Lunar Capture Transfer Using the Fuzzy Boundary and Solar Perturbations: a Survey", In: Proceedings for the International Congress of SETI Sail and Astrodynamics, Turin, Italy, 1992.

Belbruno, E.A., "The Dynamical Mechanism of Ballistic Lunar Capture Transfers in the Four-Body Problem from the Perspective of Invariant Manifolds and Hill's Regions", Institut D'Estudis Catalans, CRM Research Report No 270, (November 1994).

Belbruno, E.A., Miller, J.K., "A Ballistic Lunar Capture Trajectory for Japanese Spacecraft Hiten", Jet Propulsion Lab., JPL IOM 312/90.4-1731, Internal Document, Pasadena, CA, Jun. 1990.
Belbruno, E.A., Miller, J.K., "Sun-Perturbed Earth-to-Moon Transfers With Ballistic Capture, Journal of Guidance, Control and Dynamics, Vol. 16, no 4, (July-August 1993), pp. 770-775.

Kawaguchi, J., "On the Weak Stability Boundary Utilization and its Characteristics", AAS paper 00-176, AAS/AIAA Spaceflight Mechanics Meeting, 2000.

Krish, V., "An Investigation Into Critical Aspects of a New Form of Low Energy Lunar Transfer, the Belbruno-Miller Trajectories", Master's Dissertation, Massachusetts Institute of Technology, Cambridge, MA, Dec 1991.

Krish, V., Belbruno, E.A., Hollister, W.M., "An Investigation Into Critical Aspects of a New Form of Low Energy Lunar Transfer, the Belbruno-Miller Trajectories", AIAA paper 92-4581-CP, 1992.

Miller, J.K., Belbruno, E.A., "A Method for the Construction of a Lunar Transfer Trajectory Using Ballistic Capture", AAS-91-100. In: AAS/AIAA Space Flight Mechanics Meeting, Houston, Texas, Feb. 1991.

Prado, A.F.B.A., "Numerical Study and Analytic Estimation of the Forces Acting in the Gravitational Capture," Journal of Guidance, Control and Dynamics, Vol. 25, No. 2, pp. 368-375, 2002.

Sweetser, T, "Trajectory Design for A Europa Orbiter Mission: A Plethora of Astrodynamic Challenges", Advances in Astronautical Science, Spaceflight Mechanics, Vol. 95, Paper AAS 97-174, 1997.

Vieira Neto, E. and Prado, A.F.B.A., "A Study of the Gravitational Capture in the Restricted-Problem", Proceedings of the "International Symposium on Space Dynamics pg. 613-622. Toulouse, France, 1923/June/1995.

Vieira Neto, E. and Prado, A.F.B.A., "Study of the Gravitational Capture in the Elliptical Restricted Three-Body Problem", Proceedings of the "International Symposium on Space Dynamics pg. 202-207. Gifu, Japan, 19-25/May/1996.

Vieira Neto, E. and Prado, A.F.B.A., "Time-of-Flight Analyses for the Gravitational Capture Maneuver", Journal of Guidance, Control and Dynamics, Vol. 21, No. 1, pp. 122-126, 1998.

Vieira Neto, E., "Estudo Numérico da Captura Gravitacional Temporária Utilizando o Problema Restrito de Três Corpos", Ph.D. Dissertation, Instituto Nacional de Pesquisas Espaciais, Brazil, 1999.

Yamakawa, H., 1992, "On Earth-Moon Transfer Trajectory with Gravitational Capture”, Ph.D. Dissertation, University of Tokyo.

Yamakawa, H., Kawaguchi, J., Ishii, N., Matsuo, H., "A Numerical Study of Gravitational Capture Orbit in Earth-Moon System", AAS paper 92-186, AAS/AIAA Spaceflight Mechanics Meeting, Colorado Springs, Colorado, 1992.

Yamakawa, H., Kawaguchi, J., Ishii, N., Matsuo, H., "On Earth-Moon transfer trajectory with gravitational capture", AAS paper 93-633, AAS/AIAA Astrodynamics Specialist Conference, Victoria, Canada, 1993.

Szebehely, V.G., "Theory of Orbits", Academic Press, New York, 1967. 